\title{
Non-monochromatic Triangles in a 2-Edge-Coloured Graph
}

\author{
Matt DeVos* \\ Department of Mathematics \\ Simon Fraser University, Burnaby, B.C., Canada \\ mdevos@sfu.ca \\ Jessica McDonald ${ }^{\dagger}$ \\ Department of Mathematics and Statistics \\ Auburn University, Auburn, AL, U.S.A. \\ mcdonald@auburn.edu \\ Amanda Montejano \\ UMDI Facultad de Ciencias \\ UNAM Juriquilla, Querétaro, Mexico \\ amandamontejano@ciencias. unam.mx
}

Submitted: Sep 19, 2018; Accepted: Dec 31, 2018; Published: Jul 5, 2019

(C) The authors. Released under the CC BY-ND license (International 4.0).

\begin{abstract}
Let $G=(V, E)$ be a simple graph and let $\{R, B\}$ be a partition of $E$. We prove that whenever $|E|+\min \{|R|,|B|\}>\left(\begin{array}{c}|V| \\ 2\end{array}\right)$, there exists a subgraph of $G$ isomorphic to $K_{3}$ which contains edges from both $R$ and $B$. If instead equality holds, and $G$ has no such subgraph, then we show that $G$ is in one of a few simple classes.
\end{abstract}

Mathematics Subject Classifications: 05C15, 05C55

\section{Introduction}

Throughout all graphs are assumed to be simple. A triangle in a graph $G$ is a subgraph of $G$ isomorphic to a complete graph on 3 vertices. One of the first theorems in extremal graph theory is the following best possible result.

Theorem 1 (Mantel [7]). If $G=(V, E)$ satisfies $|E|>\frac{1}{4}|V|^{2}$, then $G$ contains a triangle.

In this paper we are interested in graphs equipped with edge colourings. We define a $k$ edge-coloured graph to be a graph $G=(V, E)$ equipped with a disjoint union $E=\sqcup_{i=1}^{k} E_{i}$ called a colouring. Every subgraph $H \subseteq G$ we will view as a $k$-edge-coloured graph

*Supported in part by an NSERC Discovery Grant (Canada).

${ }^{\dagger}$ Supported in part by NSF grant DMS-1600551.

${ }^{\ddagger}$ Supported by CONACyT 219827, DGAPA: PASPA and PAPIIT IN116519. 


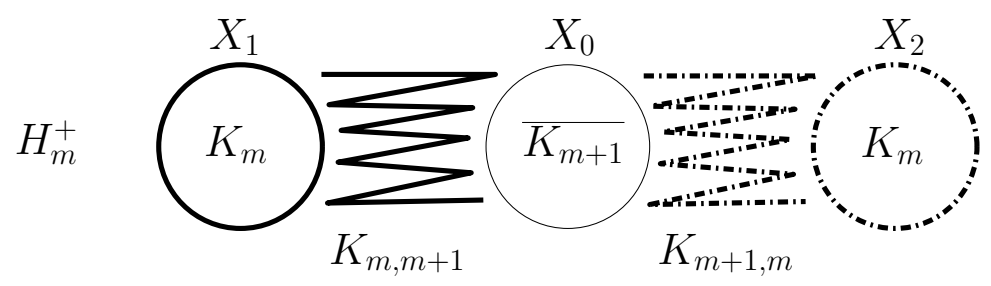

Figure 1: The graph $H_{m}^{+}$, with two colour classes indicated by the bold solid lines and bold dashed-dotted lines. Obtain $H_{m}$ from $H_{m}^{+}$by deleting a vertex in $X_{0}$.

equipped with the colouring $E(H)=\sqcup_{i=1}^{k}\left(E_{i} \cap E(H)\right)$. We say that $G$ is monochromatic if there exists $1 \leqslant i \leqslant k$ so that $E=E_{i}$, and otherwise it is non-monochromatic. If $\left|E_{i}\right| \leqslant 1$ holds for every $1 \leqslant i \leqslant k$ we call $G$ rainbow.

One of the central problems in Ramsey Theory is to determine for every $k$ and $t$ the smallest integer $n$ so that every $k$-edge-coloured $K_{n}$ contains a complete subgraph of order $t$ which is monochromatic. On the flip side, problems in Anti-Ramsey Theory are concerned with conditions forcing the existence of rainbow subgraphs. See [2] and [5] for recent surveys of Ramsey Theory and Anti-Ramsey theory, respectively. It is worth noting that Gallai has given a precise description of all possible edge-colourings of a complete graph that do not have a rainbow triangle (see $[3,6]$ ). The existence of subgraphs with a prescribed color pattern in 2-edge colorings of the complete graph has also been considered in $[1,4]$. Our problem is another variation on these themes. Here we are focused on conditions on an arbitrary graph forcing the existence of a non-monochromatic triangle. We prove the following theorem and immediate corollary.

Theorem 2. If $G=(V, E)$ is a 2-edge-coloured graph with colouring $E=E_{1} \sqcup E_{2}$, and $|E|+\min \left\{\left|E_{1}\right|,\left|E_{2}\right|\right\}>\left(\begin{array}{c}|V| \\ 2\end{array}\right)$, then $G$ contains a non-monochromatic triangle.

Corollary 3. If $G=(V, E)$ is a 2-edge-coloured graph with colouring $E=E_{1} \sqcup E_{2}$ and $\left|E_{1}\right|,\left|E_{2}\right|>\frac{1}{3}\left(\begin{array}{c}|V| \\ 2\end{array}\right)$, then $G$ contains a non-monochromatic triangle.

Theorem 2 is tight in the sense that there exist graphs missing the bound by one edge for which there is no non-monochromatic triangle (i.e. all triangles are monochromatic). We have characterized these graphs and we introduce them next. For this discussion, we need to extend the usual notion of isomorphism to coloured graphs: If $G=(V, E)$ and $G^{\prime}=\left(V^{\prime}, E^{\prime}\right)$ are $k$-edge-coloured graphs relative to the colourings $E=\sqcup_{i=1}^{k} E_{i}$ and $E^{\prime}=\sqcup_{i=1}^{k} E_{i}^{\prime}$, then we say that $G$ and $G^{\prime}$ are isomorphic if there is an isomorphism from $G$ to $G^{\prime}$ that maps every $E_{i}$ to some $E_{j}^{\prime}$.

For every positive integer $m$, define the 2-edge-coloured graph $H_{m}$ as follows. We let $V\left(H_{m}\right)=\sqcup_{i=0}^{2} X_{i}$ where $\left|X_{i}\right|=m$ for $0 \leqslant i \leqslant 2$. We add to $H_{m}$ all edges between $X_{0}$ and $X_{1} \cup X_{2}$ and add a clique on $X_{1}$ and a clique on $X_{2}$. We equip $H_{m}$ with a 2-edge-colouring by defining $E_{i}$ to be all edges incident with a vertex in $X_{i}$ for $i=1,2$. We let $H_{m}^{+}$be the 2-edge-coloured graph defined exactly as above with the exception that $\left|X_{0}\right|=m+1$ (see Figure 1). As we prove, the only cases where $|E|+\min \left\{\left|E_{1}\right|,\left|E_{2}\right|\right\}=\left(\begin{array}{c}|V| \\ 2\end{array}\right)$ for which no non-monochromatic triangle exists are: 


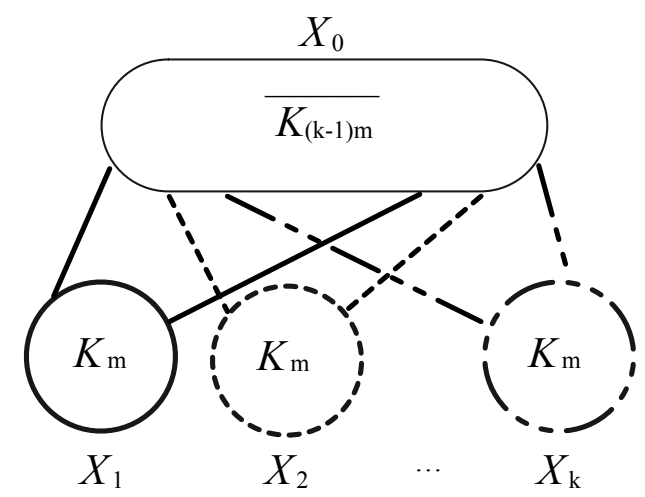

Figure 2: The graph $H_{m}^{k}$ with $k$ color clases. It indicates that Conjecture 4 is best possible.

1. $G$ is a monochromatic complete graph.

2. $G$ is a 4-cycle and $E_{i}$ is a perfect matching for $i=1,2$

3. $G$ is isomorphic to either $H_{m}$ or $H_{m}^{+}$for some $m \geqslant 1$.

Note that apart from monochromatic complete graphs, these examples satisfy $\left|E_{1}\right|=$ $\left|E_{2}\right|=\frac{1}{3}\left(\begin{array}{c}|V| \\ 2\end{array}\right)$ showing that Corollary 3 is also tight.

We conjecture the following multi-colour variations to Theorem 2 and Corollary 3.

Conjecture 4. If $G=(V, E)$ is a $k$-edge-coloured graph with colouring $E=\sqcup_{i=1}^{k} E_{i}$ and $2|E|-\max \left\{\left|E_{i}\right|: 1 \leqslant i \leqslant k\right\}>\frac{1}{2}|V|^{2}$ then $G$ contains a non-monochromatic triangle.

Conjecture 5. If $G=(V, E)$ is a $k$-edge-coloured graph with colouring $E=\sqcup_{i=1}^{k} E_{i}$ and $\left|E_{i}\right|>\frac{1}{4 k-2}|V|^{2}$ holds for $1 \leqslant i \leqslant k$, then $G$ contains a non-monochromatic triangle.

If true, these conjectures are essentially best possible thanks to a generalization of the graph $H_{m}$. For positive integers $k \geqslant 1$ and $m \geqslant 1$ define the $k$-edge-coloured graph $H_{m}^{k}$ as follows. The vertex set of $H_{m}^{k}$ is the disjoint union of sets $X_{0}, \ldots, X_{k}$ where $\left|X_{0}\right|=(k-1) m$ and $\left|X_{i}\right|=m$ for $1 \leqslant i \leqslant m$. Add to $H_{m}$ all edges between $X_{0}$ and $\cup_{i=1}^{k} X_{i}$ and add a clique on $X_{i}$ for $1 \leqslant i \leqslant k$. Equip $H_{m}^{k}$ with a $k$-edge-colouring by defining $E_{i}$ to be all edges incident with a vertex of $X_{i}$ for $1 \leqslant i \leqslant k$ (see Figure 2 ). Now $H_{m}^{k}$ does not have a non-monochromatic triangle, but $\left|V\left(H_{m}^{k}\right)\right|=(2 k-1) m$ and every $E_{i}$ satisfies

$$
\left|E_{i}\right|=\left(\begin{array}{c}
m \\
2
\end{array}\right)+(k-1) m^{2} \approx\left(k-\frac{1}{2}\right) m^{2}=\frac{1}{4 k-2}\left|V\left(H_{m}^{k}\right)\right|^{2} .
$$

One might expect a stronger form of Conjecture 4 to hold under the weaker assumption $2|E|-\max \left\{\left|E_{i}\right|: 1 \leqslant i \leqslant k\right\}>\left(\begin{array}{c}|V| \\ 2\end{array}\right)$ (indeed we originally did!). However, Sophie Spirkl noted that a rainbow 4-cycle is a counterexample to this [8]. 


\section{The Proof}

For a graph $G=(V, E)$ we let $\alpha(G)$ denote the size of the largest independent set. We define the density of $G$ to be:

$$
d(G)=\frac{|E|}{\left(\begin{array}{c}
|V| \\
2
\end{array}\right)} .
$$

We have introduced the concept of density in part because we find it easiest to think in these terms. However, it is used somewhat sparingly in the proof because it is usually easier to write out our bounds in terms of edges. The proof of our main theorem calls on the following straightforward lemma.

Lemma 6. If $G$ is a graph with $|V(G)| \geqslant 2$ and density at least $\frac{2}{3}$, there is a component $G^{\prime}$ of $G$ with $\left|V\left(G^{\prime}\right)\right|>\frac{2}{3}|V(G)|$, and furthermore,

$$
\alpha\left(G^{\prime}\right)+1 \leqslant 2\left|V\left(G^{\prime}\right)\right|-|V(G)| .
$$

Proof. Since $G$ must have a vertex with at least $\frac{2}{3}(|V(G)|-1)$ neighbours, it follows that there is a component $G^{\prime}$ of $G$ containing more than $\frac{2}{3}|V(G)|$ vertices. Define $a=\alpha\left(G^{\prime}\right)$, let $b=\left|V\left(G^{\prime}\right)\right|-a$ and $c=|V(G)|-\left|V\left(G^{\prime}\right)\right|$, and note that $\left|V\left(G^{\prime}\right)\right| \geqslant \frac{2}{3}|V(G)|$ implies $a+b \geqslant 2 c$. Also note that $b>0$ since otherwise the component $G^{\prime}$ would be forced to be an isolated vertex. Assume (for a contradiction) that $\alpha\left(G^{\prime}\right) \geqslant 2\left|V\left(G^{\prime}\right)\right|-|V(G)|$, and note that this implies $c \geqslant b$. Together with the bound $a+b \geqslant 2 c$ we then have $a \geqslant c \geqslant b$. But this gives the contradiction

$$
|E(G)| \leqslant\left(\begin{array}{l}
b \\
2
\end{array}\right)+\left(\begin{array}{c}
c \\
2
\end{array}\right)+a b<\frac{2}{3}\left(\left(\begin{array}{c}
a \\
2
\end{array}\right)+\left(\begin{array}{l}
b \\
2
\end{array}\right)+\left(\begin{array}{c}
c \\
2
\end{array}\right)+a b+a c+b c\right)=\frac{2}{3}\left(\begin{array}{c}
|V(G)| \\
2
\end{array}\right) .
$$

We need a little additional notation before proving the main result. Let $G=(V, E)$ be a 2-edge-coloured graph with colouring $E=R \sqcup B$. If $X \subseteq V$ we let $G[X]$ denote the 2-edge-coloured subgraph of $G$ induced by $X$. We let $E(X)$ denote the set of edges with both ends in $X$, and define:

$$
e(X)=|E(X)| \quad e_{B}(X)=|E(X) \cap B| \quad e_{R}(X)=|E(X) \cap R| .
$$

If $Y \subseteq V$ is disjoint from $X$, we let $E(X, Y)$ denote the set of edges with one end in $X$ and one end in $Y$. Define:

$$
e(X, Y)=|E(X, Y)| \quad e_{B}(X, Y)=|E(X, Y) \cap B| \quad e_{R}(X, Y)=|E(X, Y) \cap R| .
$$

Next we prove our main theorem, restated to allow for $|E|+\min \{|R|,|B|\} \geqslant\left(\begin{array}{c}|V| \\ 2\end{array}\right)$.

Theorem 7. Let $G=(V, E)$ be a 2-edge-coloured graph with colouring $E=R \sqcup B$. If $|E|+\min \{|R|,|B|\} \geqslant\left(\begin{array}{c}|V| \\ 2\end{array}\right)$, then one of the following holds:

1. G has a non-monochromatic triangle.

2. $G$ is a monochromatic clique. 
3. $G$ is a 4-cycle and $R, B$ are perfect matchings.

4. $G$ is isomorphic to either $H_{m}$ or $H_{m}^{+}$for some $m \geqslant 1$.

Proof. Suppose (for a contradiction) that the theorem is false, and let $G=(V, E)$ be a counterexample with $|V|$ minimum. Note that this implies $G$ is connected. We call edges in $R$ red and edges in $B$ blue, and for a vertex $v \in V$ an adjacent vertex is called a red (blue) neighbour if it is joined to $v$ by a red (blue) edge. We say that a subset $X \subseteq V$ with $|X|=3$ is a seagull if $X$ is the vertex set of a non-monochromatic two edge path $P$. Note that since $G$ does not have a non-monochromatic triangle, the subgraph $P$ must be induced. We say that a subset $X \subseteq V$ with $|X|=4$ is an alternating square if $X$ is the vertex set of a properly coloured 4-cycle $C$ (i.e. every $x \in X$ is incident with one edge in $R \cap E(C)$ and one in $B \cap E(C))$. Again in this case the subgraph $C$ must be induced. We begin with a couple of straightforward claims about the behaviour of $G$.

Claim 8. $d(G) \geqslant \frac{2}{3}$ and if $d(G)=\frac{2}{3}$, then $|R|=|B|$.

Proof of Claim. This follows from $|E|+\frac{1}{2}|E| \geqslant|E|+\min \{|R|,|B|\} \geqslant\left(\begin{array}{c}|V| \\ 2\end{array}\right)$.

Claim 9. If $X$ is a seagull in $G$ and $v \in V \backslash X$, then $e(v, X) \leqslant 2$.

Proof of Claim. If $v$ has an edge to each of the three vertices in $X$ then, regardless of their colours, we get a non-monochromatic triangle.

Claim 10. $G$ does not have an alternating square.

Proof of Claim. Suppose (for a contradiction) that $X \subseteq V$ is an alternating square. Note that $X \neq V$ since in this case $G$ satisfies the third outcome of the theorem. Define $Y=V \backslash X$ and note that by Claim 9 , every $y \in Y$ must satisfy $e(y, X) \leqslant 2$. In particular, this implies

$$
e(X, Y) \leqslant \frac{1}{2}|X||Y|
$$

It follows from (1) that

$$
\begin{aligned}
e(X)+e(X, Y)+\max \left\{e_{B}(X)+e_{B}(X, Y), e_{R}(X)+e_{R}(X, Y)\right\} \\
\leqslant 4+\frac{1}{2}|X||Y|+\left(2+\frac{1}{2}|X||Y|\right)=\left(\begin{array}{c}
|X| \\
2
\end{array}\right)+|X||Y| .
\end{aligned}
$$

We claim that $e(Y)+\min \left\{e_{R}(Y), e_{B}(Y)\right\} \geqslant\left(\begin{array}{c}|Y| \\ 2\end{array}\right)$. If not, then suppose, without loss of generality, that $e(Y)+e_{R}(Y)<\left(\begin{array}{c}|Y| \\ 2\end{array}\right)$. Equation (2) implies that $e(X)+e(X, Y)+$ $e_{R}(X)+e_{R}(X, Y) \leqslant\left(\begin{array}{c}|X| \\ 2\end{array}\right)+|X||Y|$. Summing this and $e(Y)+e_{R}(Y)<\left(\begin{array}{c}|Y| \\ 2\end{array}\right)$ give us $|E|+|R|<\left(\begin{array}{c}|V| \\ 2\end{array}\right)$ which contradicts our primary assumption.

Hence $e(Y)+\min \left\{e_{R}(Y), e_{B}(Y)\right\} \geqslant\left(\begin{array}{c}|Y| \\ 2\end{array}\right)$, and by the minimality of $G$, the theorem holds for $G[Y]$. Thus, $G[Y]$ must be either a monochromatic clique, an alternating square, or $G[Y]$ is isomorphic to one of $H_{m}$ or $H_{m}^{+}$for some $m \geqslant 1$. In all but the first case, the density of $G[Y]$ is equal to $\frac{2}{3}$, and since the density of $G[X]$ is also $\frac{2}{3}$, inequality (1) implies 

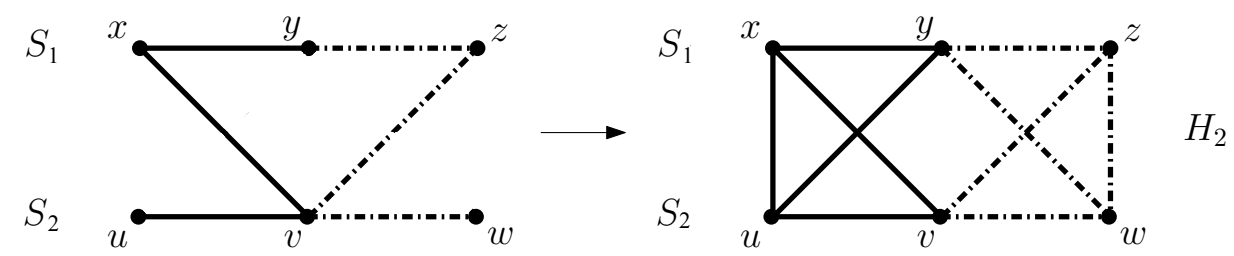

Figure 3: Forcing a copy of $\mathrm{H}_{2}$ in the proof of Claim 11. Here and elsewhere in the paper, solid bold lines indicate colour class $R$, and dashed-dotted bold lines indicate colour class $B$.

that $d(G)<\frac{2}{3}$, contradicting Claim 8. In the remaining case, $G[Y]$ is a clique and we may assume, without loss of generality, that all edges of $G[Y]$ are in $R$. Since $|E|+|B| \geqslant\left(\begin{array}{c}|V| \\ 2\end{array}\right)$ it must be that $e(X, Y)=e_{B}(X, Y)=\frac{1}{2}|X||Y|$ and every $y \in Y$ is incident with exactly two vertices in $X$. If $|Y| \geqslant 3$, then there are two vertices in $Y$ with a common neighbour in $X$ and this triple forms a non-monochromatic triangle - a contradiction. Otherwise, $1 \leqslant|Y| \leqslant 2$ and $e_{R}(X, Y)=0$ give us a contradiction to the hypothesis $|E|+|R|<\left(\begin{array}{c}|V| \\ 2\end{array}\right)$.

Claim 11. Let $S_{1}, S_{2}$ be disjoint seagulls in $G$. Let $r=e_{R}\left(S_{1}, S_{2}\right)$ and $b=e_{B}\left(S_{1}, S_{2}\right)$, then

- $r+b \leqslant 6$,

- if $r+b=6$ then $G\left[S_{1} \cup S_{2}\right]$ is isomorphic to $H_{2}$, and

- $r+b+\max \{r, b\} \leqslant 9$.

Proof of Claim. By Claim 9, there are at most 2 edges between a seagull and any other vertex, so in particular there are at most 6 edges between $S_{1}$ and $S_{2}$. If $r+b=6$ then each vertex in $S_{1}$ has exactly 2 neighbours in $S_{2}$. Label the vertices of $S_{1}$ and $S_{2}$ as $x, y, z$ and $u, v, w$ respectively, with the edges $u v$ and $x y$ being in $R$, and the edges $v w$ and $y z$ being in $B$. Observe that a blue neighbor of $v$ cannot be a neighbor of $u$, and a red neighbor of $v$ cannot be a neighbor of $w$. Hence, if each of $u, v$ and $w$ has exactly 2 neighbours in $S_{1}$, it must be that $v$ has exactly one red neighbor and one blue neighbor in $S_{1}$. Moreover, the red neighbour of $v$ in $S_{1}$ must be $x$ and the blue neighbour of $v$ in $S_{1}$ must be $z$ (otherwise, $v, x, y, z$ would induce alternating square contradicting Claim 10). This forces $G\left[S_{1} \cup S_{2}\right]$ to be $H_{2}$ (see Figure 3). Note that the third instance of the claim is satisfied in this case. It remains to prove the third instance of the claim when $r+b \leqslant 5$. For that, it suffices to show that $\max \{r, b\} \leqslant 4$. Suppose (for a contradiction, and without loss of generality) that $b \geqslant 5$. Then, there are at least three $B$-edges between $\{u, v\}$ and $S_{1}$. Avoiding the existence of non-monochromatic triangles, the two possibilities for this to happen are depicted in Figure 4. In either case, we have created an alternating square, a contradiction to Claim 10. 

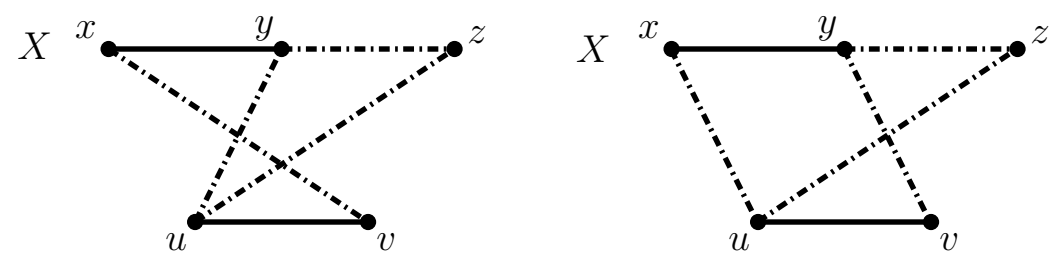

Figure 4: The two cases to consider in Claim 11 when we assume that $b \geqslant 5$.

Choose a maximum size list of vertex disjoint seagulls $S_{1}, \ldots, S_{m}$ in $G$ and define the sets $S=\cup_{i=1}^{m} S_{i}$ and $T=V \backslash S$. By Claim 9, each vertex in $T$ is adjacent to at most two of the three vertices in each seagull $S_{i}$. Hence,

$$
e(S, T) \leqslant \frac{2}{3}|S||T|
$$

Claim 12. The set $S$ satisfies

$$
\frac{3}{2} e(S) \leqslant e(S)+\max \left\{e_{R}(S), e_{B}(S)\right\} \leqslant\left(\begin{array}{c}
|S| \\
2
\end{array}\right) .
$$

Moreover, if $e(S)=\frac{2}{3}\left(\begin{array}{c}|S| \\ 2\end{array}\right)$, then $G[S]$ is isomorphic to $H_{m}$.

Proof of Claim. Since $e(S)+\frac{1}{2} e(S) \leqslant e(S)+\max \left\{e_{R}(S), e_{B}(S)\right\}$, the first inequality in (4) is immediate. The second inequality in (4) follows from the third instance of Claim 11, which states that for any distinct $S_{i}, S_{j}$ we have

$$
e\left(S_{i}, S_{j}\right)+\max \left\{e_{R}\left(S_{i}, S_{j}\right), e_{B}\left(S_{i}, S_{j}\right)\right\} \leqslant 9=\left|S_{i}\right| \cdot\left|S_{j}\right|,
$$

and from the fact that for every $S_{i}, G\left[S_{i}\right]$ has exactly one edge in $R$ and one edge in $B$, and $2+1=\left(\begin{array}{l}3 \\ 2\end{array}\right)$. Finally, if $e(S)=\frac{2}{3}\left(\begin{array}{c}|S| \\ 2\end{array}\right)$ then $e\left(S_{i}, S_{j}\right)=6$ for any $i \neq j$. Thus, by the second instance of Claim 11, G[S $\left.\cup S_{i}\right]$ is isomorphic to $H_{2}$ for any distinct $S_{i}, S_{j}$. This implies that $G[S]$ isomorphic to $H_{m}$.

For a given $x \in V$ we will use $N_{B}(x)$, and $N_{R}(x)$, to denote the set of blue neighbours of $x$, and the set of red neighbours of $x$, respectively.

Claim 13. $S \neq \emptyset$ and $|T| \geqslant 3$.

Proof of Claim. If $S=\emptyset$, then the assumption that $G$ is connected implies that one of $R$ or $B$ must be empty, and then the only way for $G$ to satisfy $|E|+\min \{|R|,|B|\} \geqslant\left(\begin{array}{c}|V| \\ 2\end{array}\right)$ is for $G$ to be a monochromatic clique. Therefore $m \geqslant 1$.

Note that, if $|T| \leqslant 2$ the only way for $G$ to satisfy Claim 8 is to have equalities in both (3) and (4), that is

$$
e(S, T)=\frac{2}{3}|S||T| \text { and } e(S)=\frac{2}{3}\left(\begin{array}{c}
|S| \\
2
\end{array}\right),
$$

and furthermore, if $|T|=2$, we must have $e(T)=1$. The second identity in (6) implies that $G[S]$ is isomorphic to $H_{m}$ (by Claim 12). So, we may write $V(G[S])=X_{0} \sqcup X_{1} \sqcup X_{2}$ as in Figure 1 where the edges incident with a vertex in $X_{1}$ are red and those incident with a vertex in $X_{2}$ are blue. 
- If $|T|=0$ then $G=G[S]$ is isomorphic to $H_{m}$, a contradiction.

- If $|T|=1$, say $T=\{v\}$. In this case we have $d(G)=\frac{2}{3}$, thus $|R|=|B|$ (by Claim 8). Since $G[S]$ is isomorphic to $H_{m}$, and $e_{R}\left(H_{m}\right)=e_{B}\left(H_{m}\right)$ then we must have $e_{R}(v, S)=e_{B}(v, S)=m$. First observe that $v$ cannot have a neighbour $x \in X_{0}$ : If say $v x \in R$, then $N_{B}(v) \subset X_{0} \backslash\{x\}$ is too small. It follows from this and Claim 10 that $N_{R}(v)=X_{1}$ and $N_{B}(v)=X_{2}$, so $G$ is isomorphic to $H_{m}^{+}$, a contradiction.

- If $|T|=2$, say $T=\{u, v\}$, and suppose without loss of generality that $u v \in R$. By (6) we have $e(S, T)=\frac{4}{3}|S|=4 m$, so both vertices in $T$ have exactly two edges each to every seagull. Since $G[S]$ is isomorphic to $H_{m}$, in order to satisfy $|E|+\min \{|R|,|B|\} \geqslant\left(\begin{array}{c}|V| \\ 2\end{array}\right)$, it must be that $e_{R}(S, T) \geqslant 2 m-1$ and $e_{B}(S, T) \geqslant 2 m$. Now if there is a vertex in $T$ with two blue edges to the same seagull, then the other vertex in $T$ has only one edge to that seagull, a contradiction. So every vertex in $T$ has at most one, hence exactly one, blue edge to every seagull, and consequently exactly one red edge to every seagull. This is imposible without creating either an alternating square or a non-monochromatic triangle.

Now we have $S \neq \emptyset$ and $|T| \geqslant 3$ and we will show that this leads to a contradiction. Note that the graph $G[T]$ must have all components monochromatic (by the maximality of our collection of seagulls). It follows from Claim 8, Equation (3), and Claim 12 that $G[T]$ has density at least $\frac{2}{3}$. Hence by Lemma $6, G[T]$ has a component $H$ with $|V(H)|>\frac{2}{3}|T|$. We shall assume (without loss) that $E(H) \subseteq R$. Next we establish a key claim concerning blue edges between our seagulls and $V(H)$.

Claim 14. For every $1 \leqslant i \leqslant m$ we have $e_{B}\left(S_{i}, V(H)\right) \leqslant \alpha(H)+1$.

Proof of Claim. To prove this claim, let $S_{i}=\{x, y, z\}$ be a seagull and assume that $x y \in R$ and $y z \in B$. Since $G$ does not have an alternating square (by Claim 10) or a nonmonochromatic triangle (by assumption) it follows that $N_{B}(x) \cap V(H)$ and $N_{B}(y) \cap V(H)$ are disjoint and moreover $\left(N_{B}(x) \cup N_{B}(y)\right) \cap V(H)$ is independent (see the left image in Figure 5). Similarly, $N_{B}(z) \cap V(H)$ is independent. If $\left|\left(N_{B}(x) \cup N_{B}(y)\right) \cap V(H)\right| \leqslant 1$ the desired bound follows immediately, and we are similarly done if $\left|N_{B}(z) \cap V(H)\right| \leqslant 1$. So we may assume both of these sets have size at least two. Choose $w \in N_{B}(z) \cap V(H)$ and (since $H$ is nontrivial) choose an edge $w w^{\prime} \in E(H)$. At most one of $w, w^{\prime}$ can appear in $\left(N_{B}(x) \cup N_{B}(y)\right) \cap V(H)$ since this set is independent. Therefore, we may choose a vertex $u \in\left(N_{B}(x) \cup N_{B}(y)\right) \cap V(H)$ with $u \neq w, w^{\prime}$. Now we have arrived at a contradiction as $\left\{z, w, w^{\prime}\right\}$ and $\{x, y, u\}$ are both seagulls (see the right image in Figure 5).

In what follows, we use $|V(H)|=h,|T|=t$, and $|S|=s$. For every seagull $S_{i}$, Claim 2 implies that $e\left(S_{i}, T\right) \leqslant 2 t$ and $e_{B}\left(S_{i}, T \backslash V(H)\right) \leqslant e\left(S_{i}, T \backslash V(H)\right) \leqslant 2(t-h)$. Using 

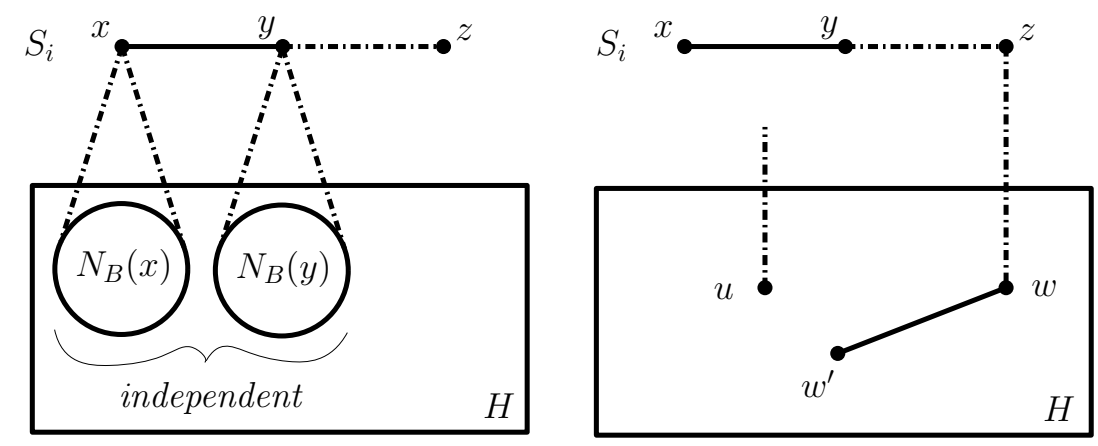

Figure 5: The situation under consideration in Claim 14.

these inequalities, together with Claim 14 and then Lemma 6, we have

$$
\begin{aligned}
e\left(S_{i}, T\right)+e_{B}\left(S_{i}, T\right) & =e\left(S_{i}, T\right)+e_{B}\left(S_{i}, V(H)\right)+e_{B}\left(S_{i}, T \backslash V(H)\right) \\
& \leqslant 2 t+\alpha(H)+1+2(t-h) \\
& \leqslant 3 t
\end{aligned}
$$

Summing this over all of our seagulls gives us $e(S, T)+e_{B}(S, T) \leqslant s t$. Claim 12 implies that $e(S)+e_{B}(S) \leqslant\left(\begin{array}{l}s \\ 2\end{array}\right)$. Combining these with the assumption $|E|+|B| \geqslant\left(\begin{array}{c}|V| \\ 2\end{array}\right)$ we deduce that

$$
e(T)+e_{B}(T) \geqslant\left(\begin{array}{l}
t \\
2
\end{array}\right) .
$$

Since $e(V(H), T \backslash V(H))=0$, we know that $\left(\begin{array}{l}t \\ 2\end{array}\right)-e(T) \geqslant h(t-h)$, and hence (9) says that $e_{B}(T) \geqslant h(t-h)$. On the other hand, if $h \neq t$, then the fact that $h \geqslant t-h$ (by Lemma 6) implies that $e_{B}(T) \leqslant\left(\begin{array}{c}t-h \\ 2\end{array}\right)<(t-h)^{2} \leqslant h(t-h)$. Therefore, we must have $V(H)=T$ and $e_{B}(T)=0$. Now the only way for inequality (9) to hold is for $H$ to be a clique; in particular this means $\alpha(H)=1$. Since $t \geqslant 3$ (by Claim 13) we can return to (7) and improve upon (8):

$$
e\left(S_{i}, T\right)+e_{B}\left(S_{i}, T\right) \leqslant 2 t+2<3 t .
$$

Summing over all of our seagulls gives us $e(S, T)+e_{B}(S, T)<s t$. Combining this with the bound $e(S)+e_{B}(S) \leqslant\left(\begin{array}{c}|S| \\ 2\end{array}\right)$ (from Claim (12)) gives us the contradiction

$$
|E|+|B|=e(S)+e_{B}(S)+e(S, T)+e_{B}(S, T)+\left(\begin{array}{c}
t \\
2
\end{array}\right)<\left(\begin{array}{c}
|V| \\
2
\end{array}\right)
$$

and this completes the proof.

\section{References}

[1] Y. Caro, A. Hansberg, A. Montejano. Unavoidable chromatic patterns in 2-colorings of the complete graph. arXiv:1810.12375.

[2] D. Conlon, J. Fox, B. Sudakov, Resent developments in graph Ramsey theory. Surveys in combinatorics 2015, London Math. Soc. Lecture Note Ser., vol. 424, Cambridge Univ. Press, Cambridge, 2015, pp. 49-118. MR 3497267 
[3] J. Fox, A. Grinshpun, J. Pach. The Erdős-Hajnal conjecture for rainbow triangles. Journal of Combinatorial Theory, Ser. B. Vol. 111, (2015), 75-125.

[4] J. Fox, B. Sudakov. Unavoidable patterns, J. Combin. Theory Ser. A. Vol. 115 (2008), no. 8, 1561-1569.

[5] S. Fujita, C. Magnant, K. Ozeki, Rainbow generalizations of Ramsey theory: a survey, Graphs. Combin. 26 (2010), no. 1. 1-30. MR 2606615

[6] A. Gyárfás, G. N. Sárközy, A. Sebő, S. Selkov. Ramsey-type results for Gallai colorings. J. Graph Theory Volume 64, Issue 3 (2010) 233-243

[7] W. Mantel, Problem 28, Wiskundige Opgaven, vol. 10, 1907, pp. 60-61.

[8] S. Spirkl. Personal communication. 